$\mathrm{J}$ o u r nal of

Mathematics

and Applications

JMA No 38, pp 71-76 (2015)

\title{
Problem with integral condition for evolution equation
}

\author{
P.I. Kalenyuk, G.Kuduk, I.V. Kohut, Z.M. Nytrebych
}

\begin{abstract}
We propose a method of solving the problem with nonhomogeneous integral condition for homogeneous evolution equation with abstract operator in a linear space $H$. For right-hand side of the integral condition which belongs to the special subspace $L \subseteq H$, in which the vectors are represented using Stieltjes integrals over a certain measure, the solution of the problem is represented in the form of Stieltjes integral over the same measure.
\end{abstract}

AMS Subject Classification: 35M10, 35M20

Keywords and Phrases: differential-symbol method, evolution equation, problem with integral condition

\section{Statement of the problem.}

The significant place in the research on problems for evolution equations in Banach spaces is taken by the semigroup theory (see, e.g., $[2,1,3,4]$ ).

In the recent years, problems with integral conditions have been intensively studied while investigating the process of diffusion of particles in a turbulent medium, processes of heat conduction, moisture transfer in capillary-porous media, problems of describing the dynamics of population abundance as well as problems of demography (see, e. g., works $[8,9,5,6,7,10]$ ).

Let $A$ be a given linear operator acting in the linear space $H$ and, for this operator, arbitrary powers $A^{n}, n=2,3, \ldots$, be also defined in $H$. We consider the problem

$$
\begin{gathered}
{\left[\frac{d}{d t}-a(A)\right] U(t)=0, \quad t \in(0 ; h),} \\
\int_{0}^{h} U(t) d t=\varphi,
\end{gathered}
$$

COPYRIGHT (C) by Publishing Department Rzeszów University of Technology P.O. Box 85, 35-959 Rzeszów, Poland 
where $\varphi \in H,(0, h) \subset \mathbb{R}, h>0, U:(0, h) \rightarrow H$ is an unknown vector-function, $a(A)$ is an abstract operator with analytical on $\Lambda \subseteq \mathbb{C}$ symbol $a(\lambda) \neq$ const.

Let $\eta(\lambda)$ be the entire function

$$
\eta(\lambda)=\frac{\exp [a(\lambda) h]-1}{a(\lambda)}
$$

and $P$ be the set of zeros of function (3). If $a\left(\lambda_{0}\right)=0$, then we assume that $\eta\left(\lambda_{0}\right)=h$. Hence, $\lambda_{0} \notin P$.

Denote by $x(\lambda)$ the eigenvector of the operator $A$, which corresponds to its eigenvalue $\lambda \in \Lambda \subseteq \mathbb{C}$, i.e. nonzero solutions in $H$ of the equations

$$
A x(\lambda)=\lambda x(\lambda), \lambda \in \Lambda \text {. }
$$

If $\lambda$ is not an eigenvalue of the operator $A$, then we assume $x(\lambda)=0$.

Consider an analytical on $\Lambda$ function

$$
a(\lambda)=\sum_{n=0}^{\infty} a_{n} \lambda^{n}
$$

which would be a symbol of the abstract operator

$$
a(A)=\sum_{n=0}^{\infty} a_{n} A^{n},
$$

in general, of infinite order, assuming that

$$
a(A) x(\lambda)=a(\lambda) x(\lambda)
$$

\section{Constructing the formal solution of the problem.}

In this section, we propose a method of solving the problem (1), (2).

Definition 1. We shall say that vector $\varphi$ from $H$ belongs $L \subseteq H$, if on $\Lambda$ there exist depending on $\varphi$ linear operator $R_{\varphi}(\lambda): H \rightarrow H, \lambda \in \Lambda$, and measure $\mu_{\varphi}(\lambda)$ such that

$$
\varphi=\int_{\Lambda} R_{\varphi}(\lambda) x(\lambda) d \mu_{\varphi}(\lambda) .
$$

Lemma 1. On the set $\Lambda^{*} \times(0, h), \Lambda^{*}=\Lambda \backslash P$, the following identity holds:

$$
\left[\frac{d}{d t}-a(A)\right]\left\{\frac{\exp [a(\lambda) t]}{\eta(\lambda)} x(\lambda)\right\} \equiv 0 .
$$

Proof. As supposed, for the operator $A$, arbitrary powers $A^{n}$, for $n=2,3, \ldots$, are defined in $H$. Then for any $\lambda \in \Lambda^{*}$ and $t \in(0, h)$ we have 


$$
\begin{aligned}
{\left[\frac{d}{d t}-a(A)\right]\left\{\frac{\exp [a(\lambda) t]}{\eta(\lambda)} x(\lambda)\right\} } & =\frac{d}{d t}\left\{\frac{\exp [a(\lambda) t]}{\eta(\lambda)} x(\lambda)\right\}-a(A)\left\{\frac{\exp [a(\lambda) t]}{\eta(\lambda)} x(\lambda)\right\} \\
& =\frac{a(\lambda) \exp [a(\lambda) t]}{\eta(\lambda)} x(\lambda)-\frac{\exp [a(\lambda) t]}{\eta(\lambda)} a(\lambda) x(\lambda) \equiv 0
\end{aligned}
$$

This completes our proof.

Theorem 1. Let in the problem (1), (2), the vector $\varphi$ belong $L$, i.e. $\varphi$ can be represented in the form (4). Then the formula

$$
U(t)=\int_{\Lambda^{*}} R_{\varphi}(\lambda)\left\{\frac{\exp [a(\lambda) t]}{\eta(\lambda)} x(\lambda)\right\} d \mu_{\varphi}(\lambda)
$$

defines a formal solution of the problem (1), (2).

Proof. According to the formulas (6), we have:

$$
\begin{aligned}
{\left[\frac{d}{d t}-a(A)\right] U(t) } & =\left[\frac{d}{d t}-a(A)\right] \int_{\Lambda^{*}} R_{\varphi}(\lambda)\left\{\frac{\exp [a(\lambda) t]}{\eta(\lambda)} x(\lambda)\right\} d \mu_{\varphi}(\lambda) \\
& =\int_{\Lambda^{*}} R_{\varphi}(\lambda)\left[\frac{d}{d t}-a(A)\right]\left\{\frac{\exp [a(\lambda) t]}{\eta(\lambda)} x(\lambda)\right\} d \mu_{\varphi}(\lambda) .
\end{aligned}
$$

From the identity (5) we obtain

$$
\left[\frac{d}{d t}-a(A)\right] U(t)=\int_{\Lambda^{*}} R_{\varphi}(\lambda)\{0\} d \mu_{\varphi}(\lambda) .
$$

Since the operator $R_{\varphi}(\lambda)$ is linear, the last integral is equal to zero, i.e. $U(t)$ formally satisfies the equality (1).

We shall prove the realization of integral condition (2) using fomula (4):

$$
\begin{aligned}
\int_{0}^{h} U(t) d t & =\int_{0}^{h}\left(\int_{\Lambda^{*}} R_{\varphi}(\lambda)\left\{\frac{\exp [a(\lambda) t]}{\eta(\lambda)} x(\lambda)\right\} d \mu_{\varphi}(\lambda)\right) d t \\
& =\int_{\Lambda^{*}} R_{\varphi}(\lambda)\left\{\int_{0}^{h} \frac{\exp [a(\lambda) t]}{\eta(\lambda)} x(\lambda) d t\right\} d \mu_{\varphi}(\lambda) \\
& =\int_{\Lambda^{*}} R_{\varphi}(\lambda)\left\{\frac{\eta(\lambda)}{\eta(\lambda)} x(\lambda)\right\} d \mu_{\varphi}(\lambda)=\int_{\Lambda^{*}} R_{\varphi}(\lambda) x(\lambda) d \mu_{\varphi}(\lambda)=\varphi .
\end{aligned}
$$

This completes our proof. 
Remark. The formula (6) defines a solutions of the problem (1), (2) just formally, since the following equalities are not justified:

$$
\begin{gathered}
{\left[\frac{d}{d t}-a(A)\right] \int_{\Lambda^{*}} R_{\varphi}(\lambda)\left\{\frac{\exp [a(\lambda) t]}{\eta(\lambda)} x(\lambda)\right\} d \mu_{\varphi}(\lambda)=} \\
=\int_{\Lambda^{*}} R_{\varphi}(\lambda)\left[\frac{d}{d t}-a(A)\right]\left\{\frac{\exp [a(\lambda) t]}{\eta(\lambda)} x(\lambda)\right\} d \mu_{\varphi}(\lambda), \\
\int_{0}^{h}\left(\int_{\Lambda^{*}} R_{\varphi}(\lambda)\left\{\frac{\exp [a(\lambda) t]}{\eta(\lambda)} x(\lambda)\right\} d \mu_{\varphi}(\lambda)\right) d t= \\
=\int_{\Lambda^{*}} R_{\varphi}(\lambda)\left\{\int_{0}^{h} \frac{\exp [a(\lambda) t]}{\eta(\lambda)} x(\lambda) d t\right\} d \mu_{\varphi}(\lambda) .
\end{gathered}
$$

We do not prove the existence of the Stieltjes integrals in the equalities (7) and (8) as well.

\section{Problem with integral condition for partial differ- ential equation.}

In this section, we shall give the example of using an abstract approach to solving the problem for the partial differential equation

$$
\begin{gathered}
{\left[\frac{\partial}{\partial t}-a\left(\frac{\partial}{\partial x}\right)\right] U(t, x)=0, \quad t \in(0 ; h), \quad x \in \mathbb{R},} \\
\int_{0}^{h} U(t, x) d t=\varphi(x), \quad x \in \mathbb{R}
\end{gathered}
$$

where $a\left(\frac{\partial}{\partial x}\right)$ is an operator generally of infinite order with entire symbol $a(\lambda) \neq$ const.

The problem (9), (10) has been studied in the work [11] by means of the differential-symbol method $[12,13]$. We shall represent this problem as problem (1), (2), in which $A=\frac{d}{d x}, \exp [\lambda x]$ is an eigenfunction of the operator $A, H$ is a class of entire functions, $L=K_{M}$ is a class of quasipolynomials

$$
\varphi(x)=\sum_{j=1}^{m} Q_{j}(x) \exp \left[\alpha_{j} x\right],
$$

where $\alpha_{j} \in M \subseteq \mathbb{C}, \alpha_{j} \neq \alpha_{k}$ for $j \neq k, x \in \mathbb{R}, m \in \mathbb{N} ; Q_{j}(x), j=\overline{1, m}$, are polynomials with complex coefficients.

As a measure $\mu(\lambda)$, take the Dirac measure. From the representation (4) we obtain

$$
\varphi(x)=\left.R_{\varphi}(\lambda) \exp [\lambda x]\right|_{\lambda=0},
$$


from which it follows that

$$
R_{\varphi}(\lambda)=\varphi\left(\frac{d}{d \lambda}\right)
$$

Each quasipolynomial $\varphi(x)$ of the form (11) defines a differential operation $\varphi\left(\frac{d}{d \lambda}\right)$ of finite order on the class of entire functions $\Phi(\lambda)$, namely

$$
\varphi\left(\frac{d}{d \lambda}\right) \Phi(\lambda)=\sum_{j=1}^{m} Q_{j}\left(\frac{d}{d \lambda}\right) \Phi\left(\lambda+\alpha_{j}\right),
$$

in particular,

$$
\left.\varphi\left(\frac{d}{d \lambda}\right) \Phi(\lambda)\right|_{\lambda=0}=\left.\sum_{j=1}^{m} Q_{j}\left(\frac{d}{d \lambda}\right) \Phi(\lambda)\right|_{\lambda=\alpha_{j}} .
$$

From formula (6), we obtain the representation of the solution of problem (9), (10) in the form

$$
U(t, x)=\left.\varphi\left(\frac{d}{d \lambda}\right)\left\{\frac{\exp [a(\lambda) t+\lambda x]}{\eta(\lambda)}\right\}\right|_{\lambda=0},
$$

moreover, this solution exists and is unique in appropriate class of quasipolynomials of variables $t, x$, if at that $\varphi \in K_{M}$, where $M=\mathbb{C} \backslash P, P$ is the set of zeros of function $(3)$.

Conclusions. In this work, we propose a method of solving a problem with nonhomogeneous integral condition for homogeneous evolution equation with abstract operator in a linear space. The solution of the problem is represented in the form of Stieltjes integral over a certain measure. We give the example of applying this method to solving the problem with integral condition for partial differential equation.

In the future research, the subject of interest is the development of analogous method of solving the problem for nonhomogeneous evolution equation.

\section{References}

[1] Hille E., Phillips R.J. Functional analysis and semigroups, Amer. Math. Soc., 1982, Vol. 31, 820 p.

[2] Krein S. Linear differential equations in Banach space, Amer. Math. Soc., 1971, Vol. 29, 395 p.

[3] Pazy A. Semigroups of linear operators and applications to partial differential equations, New York: Springer-Verlag, 1983, 287 p.

[4] Yosida K. Functional analysis, New York: Springer-Verlag, 1980, 513 p.

[5] Ionkin N. I. Solving a boundary value problem in heat conduction theory with nonlocal boundary conditions, Diff. equations, 1977, Vol. 13, No 2, p. 294-304. (in Russian). 
[6] Fardigola L. V. Integral boundary value problem in a strip, Math. notes, 1993, Vol. 53, No 6. p. 122-129. (in Russian).

[7] Pulkina L. S. Nonlocal problem with integral conditions for hyperbolic equation, Diff. equations, 2004, Vol. 40, No 7, p. 887-892.

[8] Cannon J. R. The solution of the heat equation. Subject to the specification of energy, Quart. Appl. Math., 1963, Vol. 21, p. 155-160.

[9] Cannon J. R., Rundell W. An inverse problem for an elliptic partial differential equation, J. Math. Anal. Appl., 1987, Vol. 126, p. 329-340.

[10] Bouziani A. Initial boundary-value problems for a class of pseudoparabolic equations with integral boundary conditions, J. Math. Anal. Appl., 2004, Vol. 291, p. $371-386$.

[11] Kalenyuk P. I., Kohut I. V., Nytrebych Z. M. Problem with integral condition for a partial differential equation of the first order with respect to time, J. Math. Sci., 2012, Vol. 181, No 3, p. 293-304.

[12] Kalenyuk P. I., Baranetskyi Ya. Ye., Nytrebych Z. N. Generalized method of separation of variables, Kyiv: Naukova dumka, 1993. 232 p. (in Russian).

[13] Kalenyuk P. I., Nytrebych Z. M. General ized scheme of separation of variables. Differential-symbol method, Lviv: Publishing house of Lviv Polytechnic National University, 2002, 292 p. (in Ukrainian).

DOI: $10.7862 / \mathrm{rf.2015.6}$

\section{P.I. Kalenyuk}

email: pkalenyuk@gmail.com

Lviv Polytechnic National University,

Lviv, Ukraine

University of Rzeszów,

Rejtana str 16 C, Rzeszow, Poland

\section{V. Kohut}

email: ikohutua@yahoo.com

Lviv Polytechnic National University

Lviv, Ukraine

\section{G.Kuduk - corresponding author} email: gkuduk@onet.eu

Wólka Niedźwiedzka 358

a graduate of the Rzesz ow University

Rejtana str 16 C, Rzeszow, Poland

\section{Z.M. Nytrebych}

email: znytrebych@gmai.com

Lviv Polytechnic National University

Lviv, Ukraine

Received 08.02.2014 